\title{
Motivational and Control Mechanisms Underlying Adolescent versus Adult Alcohol Use
}

\author{
Janna Cousijn ${ }^{1,2, *}$, Kayla H. Green ${ }^{3}{ }^{(}$, Maaike Labots ${ }^{3}$, Louk J. M. J. Vanderschuren ${ }^{3}{ }^{(1)}$, \\ J. Leon Kenemans ${ }^{4}$ and Heidi M. B. Lesscher ${ }^{3}$ \\ 1 Neuroscience of Addiction (NofA) lab, Department of Psychology, University of Amsterdam, \\ 1001NK Amsterdam, The Netherlands \\ 2 Amsterdam Brain and Cognition (ABC), University of Amsterdam, 1001NK Amsterdam, The Netherlands \\ 3 Department of Animals in Science and Society, Division of Behavioural Neuroscience, Faculty of Veterinary \\ Medicine, Utrecht University, 3584CM Utrecht, The Netherlands; green.k.h@outlook.com (K.H.G.); \\ maaikelabots@gmail.com (M.L.); L.J.M.J.Vanderschuren@uu.nl (L.J.M.J.V.); \\ H.M.B.Lesscher@uu.nl (H.M.B.L.) \\ 4 Helmholtz Research Institute, Department of Experimental Psychology, Utrecht University, \\ 3584CS Utrecht, The Netherlands; J.L.Kenemans@uu.nl \\ * Correspondence: j.cousijn@gmail.com
}

Received: 4 September 2020; Accepted: 19 September 2020; Published: 22 September 2020

\begin{abstract}
Increased motivation towards alcohol use and suboptimal behavioral control are suggested to predispose adolescents to alcohol use disorders (AUDs). Paradoxically however, most adolescent AUDs resolve over time without any formal intervention, suggesting adolescent resilience to AUDs. Importantly, studies directly comparing adolescent and adult alcohol use are largely missing. We therefore aimed to unravel the moderating role of age in the relation between alcohol use and motivational and control-related cognitive processes in 45 adolescent drinkers compared to 45 adults. We found that enhancement drinking motives and impulsivity related positively to alcohol use. Although enhancement drinking motives and impulsivity were higher in adolescents, the strength of the relation between these measures and alcohol use did not differ between age groups. None of the alcohol use-related motivational measures (i.e., craving, attentional bias, and approach bias) and behavioral control measures (i.e., interference control, risky decision making, and working-memory) were associated with alcohol use or differed between age groups. These findings support the role of impulsivity and affective sensitivity in adolescent drinking but question the moderating role of age therein. The current study contributes towards understanding the role of age in the relation between alcohol use and cognition.
\end{abstract}

Keywords: alcohol; adolescence; cognition; drinking motives

\section{Introduction}

Adolescence is marked by increases in experimentation and risk taking, positively stimulating exploration, social development, and growth towards independence [1]. Due to this very same experimentation and exploration, adolescents can also make potentially unhealthy choices, including those related to alcohol use. Indeed, the prevalence of binge drinking and alcohol use disorders (AUDs) rapidly rises over the course of adolescence towards adulthood [2-4]. Paradoxically, adolescence also poses a window of opportunity to overcome AUDs as adolescents are highly flexible [1], less sensitive to the deleterious effects of brain trauma [5], and most adolescent AUDs resolve over time without any formal intervention [2]. Since most studies into AUDs in adolescents focus on this age group only, little is known about the potentially moderating role of age in the relation between 
alcohol use and cognition. Therefore, the current study aimed to investigate the relation between motivation- and control-related cognitive functions and alcohol use in adolescents compared to adults.

Excessive alcohol use and loss of control over alcohol use, often despite awareness of its negative consequences, are considered core features of AUD [6]. Population-based studies suggest that the earlier one starts drinking, the higher the risk to develop an AUD later in life is [7-10]. Multiple cognitive mechanisms are thought to increase adolescents' propensity to develop an AUD. That is, normative reward, emotion, and social sensitivity during adolescence [11] may result in strengthened motivational drives like craving, attentional bias (i.e., allocation and maintenance of attention) and approach action tendencies in response to alcohol and alcohol cues in adolescents compared to adults. In addition, greater reinforcement learning during adolescence [12] may further facilitate the formation of substance-biased motivational processes [13], which would result in stronger motivation for alcohol use in adolescents. Moreover, behavioral control is considered to be suboptimal in adolescence, especially in emotional and arousing (social) contexts in which alcohol is generally consumed [11], which may promote escalation of alcohol use. Moreover, alcohol use is known to negatively impact adolescent cognitive development [14] and the direct detrimental pharmacological impact of alcohol on brain and cognition may be larger during adolescence (for review see [15]), supporting a stronger negative relation between drinking and cognitive control in adolescents compared to adults.

Importantly, however, most knowledge about the relation between alcohol use and cognition is based on separate studies in adolescent and adult populations. To the best of our knowledge, only three studies directly compared adolescent and adult drinkers. While McAteer et al. [16] did not find a difference in attentional bias for alcohol pictures between light-to-heavy alcohol drinking adolescents and young adults, Scheel et al. [17] showed that a low dose of alcohol, compared to placebo, negatively affected attentional flexibility in adolescents but not adults. Finally, Rooke and Hine [18] showed that alcohol-biased memory associations predicted binge drinking more strongly in adolescents than in adults. However, the lack of matching of current alcohol use and related problem severity between the adolescent and adult age groups prevent us from drawing any strong conclusions about whether the relation between alcohol use and cognition differs between adolescents and adults.

Therefore, the aim of the current study was to further explore the moderating role of age in the relation between alcohol use and cognition in 45 light-to-heavy drinking adolescents between 16 and 17 years and 45 adults between 30 and 35 years, closely matched for their monthly level of alcohol use and their alcohol use-related problems. We assessed a broad range of cognitive functions to tap into different motivational and control processes that are known to be associated with alcohol use and AUD [19,20]. Alcohol-oriented motivational processes were assessed, including measurements of alcohol craving, attentional bias, approach bias, and explicit self-reported drinking motives, next to a more general assessment of appetitive and aversive motivational sensitivity. Regarding control processes, in addition to an impulsivity self-report, various aspects of executive functioning were assessed, including measures of cognitive control, risky decision-making, and working memory. As described above, based on the theoretical framework of adolescent cognitive development, we expected adolescents to show stronger alcohol-oriented motivational processes, but weaker control processes compared to adults. Further, we expected age to moderate the relation between motivational measures and measures of alcohol use and severity of alcohol use-related problems, such that a stronger association was expected in adolescents compared to adults. Moreover, the negative relationship between control measures and alcohol use severity was expected to be more pronounced in adolescents.

\section{Materials and Methods}

\subsection{Participants}

Light to heavy drinking adolescents ( $16-17$ years; $\mathrm{n}=45 ; \mathrm{n}=23$ male) and adults ( $30-35$ years; $\mathrm{n}=48 ; \mathrm{n}=23$ male) were recruited via social media advertisements. The two age groups were closely 
matched on average self-reported alcohol consumption (standard units per month), alcohol use-related problems (Alcohol Use Disorder Identification Test [AUDIT; 21]; Figure 1), educational level and gender. To ensure an equal and homogeneous distribution of low to heavy drinkers in the adolescent and adult groups, recruitment was specifically targeted to drinking frequency (i.e., ranging from once a month to daily) and regularly adapted to complement the already included sample. Potential participants were excluded if they did not drink any alcohol, used psychotropic medication, had a history of mental illness, or did not proficiently speak Dutch. Participant eligibility was initially verified via an online survey and later confirmed during the test session, which resulted in an additional exclusion of three adult females (two due to recent pregnancy-related abstinence, one due to insufficient proficiency of the Dutch language). See Table 1 for an overview of the sample characteristics of the final sample of 45 adolescents and 45 adults.

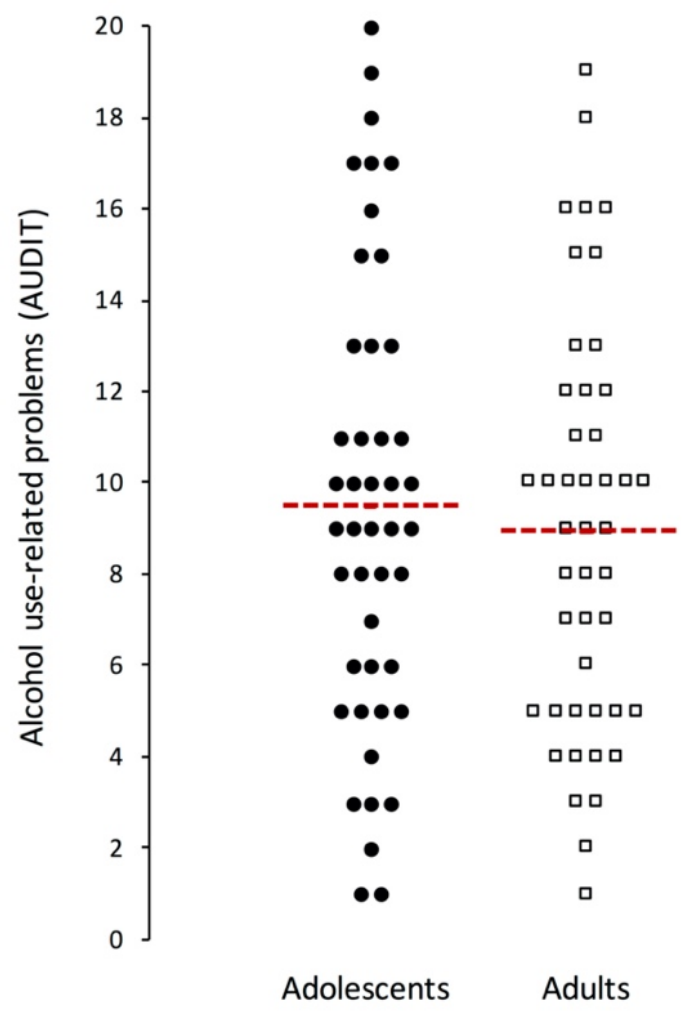

Figure 1. Distribution of alcohol use-related problems in adolescent and adult participants. Red dotted lines reflect mean Alcohol Use Disorder Identification Test (AUDIT) score for adolescents $(M=9.5)$ and adults $(\mathrm{M}=8.9)$.

\subsection{Questionnaires on Substance Use and Mental Health}

The severity of alcohol use-related problems was measured with the 10-item AUDIT [21]. The AUDIT includes 3 questions on alcohol consumption patterns and 7 questions on frequency of use-related problems, scaled from never (0) to almost daily (4) during the past year. The AUDIT is a well validated screening instrument for hazardous alcohol drinking and the two factor model seems generally supported [22]. Internal consistency of the AUDIT was acceptable in the present sample (Cronbach's alpha of 0.70 ). In addition, detailed information was collected on the history of alcohol use (i.e., age first drink, age first binge, age first time drunk, average drinking days per month, and standard drinks per drinking episode) and lifetime illicit substance use. The 6-item Fagerström Test for Nicotine Dependence (FTND) was administered to assess the severity of nicotine use disorder in daily smokers [23]. Among the 37 daily smokers in the current sample, internal consistency of the FTND was questionable (Cronbach's alpha of 0.64). To characterize mental health in the current sample, the Beck 
Depression Inventory (BDI) [24] was used to assess the presence and severity of depressive symptoms. The BDI consists of 21 questions about how the participant felt during the past week with 4 answer options (rated from 0 to 3) to indicate intensity or frequency. Internal consistency of the total BDI sum-score was good in the present sample (Cronbach's alpha of 0.80). Finally, the State-Trait Anxiety Inventory (STAI) was used to measure anxiety in two ways: as an emotional state (20 items on how one currently feels) and as a personal trait (20 items on how one generally feels) [25]. Items were rated on a 4-point Likert scale ranging from almost never (1) to almost always (4) and summed to obtain total scores. The internal consistency of the STAI was excellent in the present sample (Cronbach's alpha of 0.90 for both the STAI-state and STAI-trait scale).

Table 1. Sample Characteristics.

\begin{tabular}{|c|c|c|c|c|c|c|c|c|}
\hline & \multicolumn{3}{|c|}{ Adolescents } & \multicolumn{3}{|c|}{ Adults } & \multirow[b]{2}{*}{$p$} & \multirow[b]{2}{*}{$B F_{01}$} \\
\hline & $N$ & Mean & $S D$ & $N$ & Mean & $S D$ & & \\
\hline Age & 45 & 16.5 & 0.5 & 45 & 31.6 & 1.5 & & \\
\hline Male & 23 & & & 23 & & & & \\
\hline \multicolumn{9}{|l|}{ Alcohol use: } \\
\hline AUDIT total & 45 & 9.5 & 5.0 & 45 & 8.9 & 4.5 & 0.593 & 4.00 \\
\hline AUDIT problems (sum AUDIT 4-10) & 45 & 3.6 & 3.2 & 45 & 3.4 & 3.5 & $0.622^{+}$ & 4.04 \\
\hline Monthly use, (standard units) & 45 & 40.5 & 37.9 & 45 & 40.7 & 30.9 & $0.478^{+}$ & 3.92 \\
\hline Monthly use (days) & 45 & 6.1 & 0.7 & 45 & 12.6 & 1.2 & $0.000^{+}$ & 0.01 \\
\hline Drinks per drinking episode (standard units) & 45 & 6.3 & 0.5 & 45 & 3.3 & 0.3 & $0.000^{\dagger}$ & 0.00 \\
\hline Age first drink & 45 & 14.2 & 1.2 & 45 & 14.1 & 1.8 & $0.437^{\dagger}$ & 3.49 \\
\hline Age first binge & 43 & 15.0 & 1.1 & 45 & 16.4 & 2.5 & $0.001^{+}$ & 0.07 \\
\hline Age first time drunk & 39 & 15.0 & 0.9 & 44 & 15.8 & 2.6 & $0.047^{+}$ & 1.23 \\
\hline Nicotine dependence (FTND) & 19 & 1.4 & 1.7 & 18 & 1.7 & 2.2 & $0.964^{+}$ & 3.01 \\
\hline Lifetime Illicit substance (episodes) & 44 & 5.0 & 21.1 & 43 & 17.1 & 36.9 & $0.011^{\dagger}$ & 0.70 \\
\hline Depression (BDI) & 45 & 8.2 & 6.2 & 45 & 5.6 & 4.6 & $0.033^{\dagger}$ & 0.43 \\
\hline Anxiety (STAI): State & 45 & 32.0 & 7.3 & 45 & 34.4 & 8.8 & $0.168^{\dagger}$ & 2.50 \\
\hline Anxiety (STAI): Trait & 45 & 34.9 & 8.2 & 45 & 38.5 & 10.1 & 0.064 & 1.06 \\
\hline \multicolumn{9}{|l|}{ Motivational and Control Measures } \\
\hline Session induced alcohol craving (VAS) & 45 & 0.0 & 1.1 & 45 & 0.0 & 2.1 & $0.454^{+}$ & 3.69 \\
\hline Alcohol attentional bias (Alcohol Stroop, s) & 45 & 1.3 & 4.5 & 44 & 1.2 & 3.5 & $0.802^{\dagger}$ & 4.45 \\
\hline Cannabis-Alcohol Approach Avoidance task: & & & & & & & & \\
\hline Alcohol approach bias (ms) & 44 & -31.0 & 81.2 & 45 & -10.6 & 83.7 & 0.246 & 2.48 \\
\hline Cannabis approach bias (ms) & 44 & -20.6 & 109.8 & 45 & -17.8 & 79.1 & 0.891 & 4.47 \\
\hline Soda approach bias (ms) & 44 & -17.6 & 70.0 & 45 & -8.4 & 79.9 & 0.565 & 3.89 \\
\hline Neutral approach bias (ms) & 44 & -18.4 & 85.9 & 45 & -14.8 & 76.9 & 0.836 & 4.42 \\
\hline Drinking Motives (DMQ-r): Social & 44 & 11.6 & 5.2 & 44 & 11.8 & 4.1 & $0.957^{\dagger}$ & 4.47 \\
\hline Drinking Motives (DMQ-r): Coping & 44 & 3.8 & 4.3 & 44 & 3.4 & 3.1 & $0.836^{+}$ & 4.25 \\
\hline Drinking Motives (DMQ-r): Enhancement & 44 & 9.3 & 4.7 & 44 & 6.8 & 3.8 & $0.009^{+}$ & 0.24 \\
\hline Drinking Motives (DMQ-r): Conformity & 44 & 0.8 & 1.6 & 44 & 1.2 & 1.6 & $0.247^{\dagger}$ & 3.25 \\
\hline Impulsivity (BIS-11) & 45 & 66.1 & 11.0 & 45 & 58.6 & 8.7 & 0.001 & 0.02 \\
\hline Interference control (Classical Stroop) & 45 & 0.7 & 0.1 & 45 & 0.7 & 0.1 & $0.597^{\dagger}$ & 4.05 \\
\hline Risky decision making (CCT, cards) & 44 & 8.3 & 1.8 & 45 & 8.21 & 2.3 & 0.781 & 4.35 \\
\hline Working memory (SOPT, correct clicks) & 43 & 59.6 & 4.4 & 45 & 59.1 & 4.6 & 0.623 & 4.03 \\
\hline
\end{tabular}

SD: standard deviation; s: seconds; ms: milliseconds; AUDIT: Alcohol Use Disorder Identification Test; FTND: Fagerström Test for Nicotine Dependence; BDI: Beck Depression Inventory; STAI: State-Trait Anxiety Inventory; VAS: Visual Analogue Scale; DMQ-r: Drinking Motives Questionnaire Revised; BIS-11: Barratt Impulsiveness Scale; CCT: Colombia Card Task; SOPT: Self-ordered Pointing task; p-values reflect group comparison with independent sample t-test or non-parametric Mann-Whitney $\mathrm{U}^{\text {test }}{ }^{\dagger} ; \mathrm{BS}_{01}$ : Bayes factor likelihood $\mathrm{H} 0$ relative to $\mathrm{H} 1$ with default priors.

\subsection{Motivational and Cognitive Control Measures}

Craving: Craving for alcohol was assessed at the start and at the end of the test session with two separate Visual Analogue Scales (VAS) asking how much they craved drinking alcohol. Responses ranged from "not at all" (0) to "very much" (10) and the difference between the post-session score and the pre-session score was used as a measure of session-induced craving. 
Attentional bias: Attentional bias for alcohol-related words was measured with the paper version of the Dutch Alcohol Stroop [26]. The Alcohol Stroop involves an alcohol and a neutral subtask. The alcohol subtask consisted of a sheet of paper on which 14 alcohol-related words were each printed in blue, red, yellow, and green (52 words in total) in random order on a grey background. The neutral subtask was similar but contained 14 words related to office supplies, matched on length, number of syllabi, and frequency to the alcohol-related words. Participants were instructed to name aloud the colors in which the words were printed as quickly as possible. Subtask order was counterbalanced between participants. Time to complete each subtask was recorded in seconds with a stopwatch. The alcohol attentional bias score was calculated by subtracting the response time of the neutral subtask from the response time of the alcohol subtask, such that a positive score would indicate slower color naming for alcohol versus neutral words.

Approach bias: The joystick Cannabis-Alcohol Approach Avoidance Task (CA-AAT; see [26] for a detailed task description) was used to measure approach bias towards alcohol-related pictures. To account for general substance, appetitive and neutral approach tendencies, the CA-AAT contained a cannabis, appetitive (non-alcoholic beverages), and neutral (office supplies) control condition. During the CA-AAT, participants viewed pictures tilted $3^{\circ}$ to the left or right and were instructed to push (avoid) or pull (approach) the joystick in response to the tilt direction. Half of the participants were instructed to approach images tilted to the left and avoid images tilted to the right, while the other participants received opposite instructions. To increase approach and avoidance embodiment, pictures zoomed in during a pull response and out during a push response. The CA-AAT consisted of a cannabis and alcohol block, of which the order was counterbalanced across participants. The appetitive and neutral conditions were presented in both blocks. Each condition included 12 pictures that were presented in random order, repeated twice in pull and twice in push format, resulting in a total of 48 trials per condition. Reaction times were logged after a full push or pull response. Participants viewed a red cross after an incorrect response, after which the incorrect trial was repeated. The distance between the computer screen and the participant was held constant at $60 \mathrm{~cm}$ and the joystick was placed exactly in the middle of this distance. The alcohol, cannabis, appetitive, and control approach bias scores were calculated by subtracting the average approach reaction time from the average avoidance reaction time for each category separately. As such, a positive score would represent a bias towards faster approach relative to avoidance responses. Internal consistency of the picture bias scores per category were poor (Cronbach's alpha's: alcohol $=0.38$, cannabis $=0.51$, appetitive $=0.39$, and control $=0.33$ ), but not unusual for reaction time tests [27].

Drinking motives: The 20-item Drinking Motives Questionnaire-revised (DMQ-r) was used to assess frequency of self-reported drinking motives. The DMQ-r differentiates between four drinking motives: social, coping, enhancement, and conformity [28]. Participants were presented with 20 different statements pertaining to the four drinking motives and asked to indicate on a 5-point Likert scale, ranging from rarely/never (0) to almost always/always (4), how often they drank alcohol for that particular motive. Individual item scores were summed to obtain each drinking motive score. Internal consistency was mostly good in the present sample (Cronbach's alpha's: social $=0.84$, coping $=0.85$, enhancement $=0.81$, and conformity $=0.64$ ).

Impulsivity: Impulsiveness was measured with the 30-item Barratt Impulsiveness Scale [29]. The BIS-11 consists of 30 items describing common impulsive behaviors. Items were rated on a 4-point Likert scale ranging from rarely/never (1) to almost always/always (4). Individual item scores were summed to obtain subscale scores and a total score. Internal consistency was good (Cronbach alpha $=0.83$ ).

Interference control: The validated Dutch version of the Classical Stroop Task was used as a general measure of interference control [30]. The task consists of three subtasks with a fixed order, of which the first subtask required participants to read aloud four different color words (green, red, blue, yellow, and printed in black ink, each repeated 25 times) as fast as possible. The second subtask required participants to name the colors of 100 vertical color bars in the same four colors (green, red, 
blue, and yellow) as fast as possible. As a proxy of cognitive control, the third subtask introduced a conflict between the automatic process of word-reading and the process of color naming; participants were required to name the ink color of 100 incongruent color words (e.g., red printed in blue ink) as fast as possible. The time to complete each subtask was recorded in seconds with a stopwatch. The Stroop interference score was calculated by dividing the reaction time for the second subtask by the reaction time for the third subtask.

Risky decision making: The "hot" version of the Columbia Card Task (CCT) is a dynamic computerized card game that is sensitive to individual and developmental differences in risk-taking behavior [31]. The CCT contained 24 game-rounds during which participants were instructed to win as many points as possible by turning faced-down gain and loss cards. Each game-round started with a grid of 32 cards with varying numbers of hidden gain and loss cards. There are three game parameters: number of loss cards (1 or 3), loss amount (250 or 750 points), and gain amount (10 or 30 points). These 8 unique trials were each repeated 3 times, resulting in a total of 24 game-rounds. Participants could freely turn as many cards as they liked until they hit a loss card, after which a new game round would start. The gain and loss points were added to a total score that was visible on the top of the screen. To further motivate participants, the deceptive instruction was given that the total points of three randomly selected game rounds would be paid out in real money. Higher risky decision making corresponds to turning more cards per round, as such, the average number of turned cards across all 24 game rounds was used in subsequent analyses.

Working memory: Working memory was assessed with a computerized Self-Ordered Pointing Task [32]. The SOPT is a visual-spatial task during which participants need to remember what and where they clicked with the mouse cursor. During each trial, participants viewed a grid of different pictures and were instructed to click once on each picture. However, after each click, pictures shuffled location and participants were not allowed to click the same location twice in a row. The SOPT consisted of 8 trials with increasing difficulty; 2 trials with $6,8,10$, and 12 pictures, first with objects (e.g., flower, book, and shoe) and then repeated with more difficult abstract line drawings. Less errors would relate to better visual-spatial working memory, as such, total number of correct clicks was used in further analysis.

\subsection{Procedure}

The present study was approved by the local Ethics Committee of the Faculty of Social and Behavioral Sciences of Utrecht University. All test sessions took place at Utrecht University and at local libraries across the Netherlands. Participants provided active informed consent at the start of the test session. At least one week before the planned test session, adolescents' primary caregivers received a study information letter with the option to withdraw the adolescent's participation. The test session lasted $\sim 95 \mathrm{~min}$ and both adults and adolescents received 15 Euros for their participation.

Potential participants first filled out an online survey to screen for inclusion and exclusion criteria (e.g., age and average monthly alcohol consumption). Potential participants were subsequently contacted via email to provide all study details, verify eligibility, and to make an appointment for the test session. The test session started with the informed consent procedure, after which the participants completed the first craving assessment. This was followed by the Alcohol Stroop, Classical Stroop, CA-AAT, CCT, and SOPT. Task order was semi-random, with the classical Stroop always following the Alcohol Stroop to prevent training effects. All questionnaires were administered after the tasks and the test session was completed with the second craving assessment.

\subsection{Data Preparation and Analysis}

Due to technical issues, data from the CA-AAT was missing for one adolescent, the SOPT for 2 adolescents, and the CCT for one adolescent. Moreover, one adolescent and one adult did not fill out the DMQ. Data were inspected to check for outliers (i.e., scores deviating more than 3 standard deviations from the group mean) and the assumption of normality. One adult had an alcohol attentional 
bias score deviating 4.7 standard deviations from the mean and was therefore excluded from further analysis. Regarding the CA-AAT, error trials were also removed and reaction time data were corrected for outliers by removing reaction times below $200 \mathrm{~ms}$, above $2000 \mathrm{~ms}$, and reaction times deviating more than 3 standard deviations from the individual mean per participant [26].

To investigate differences between adolescents and adults, demographic, substance use, mental health, motivational, and control measures were first compared between groups with independent sample t-tests or non-parametric Mann-Whitney $U$ tests in case of non-normally distributed data. Regarding the CA-AAT, to investigate group differences and whether the alcohol approach bias deviated from the cannabis, appetitive and neutral approach bias, a $2 \times 4$ repeated measures ANOVA was performed with Group (i.e., adolescent and adult) as between subject factor and Bias Condition (i.e., cannabis, alcohol, appetitive, and neutral) as within subject factor.

Next, non-parametric univariate Kendall's tau rank correlations were computed to investigate the relation of alcohol use with motivational and control measures across age groups. To investigate different aspects of alcohol use, three separate outcome variables were chosen: alcohol use-related problem severity (sum AUDIT 4-10), number of drinking days per month, and number of drinks per drinking episode (standard units). Finally, a series of hierarchical regression analyses were conducted to investigate the potential moderating role of age group in the relationship between alcohol use and cognition. Simple moderation analyses were run separately for the three alcohol outcome measures and only run for those motivational and control measures that either differed between age groups (i.e., significant main effect age) or correlated with any of the three alcohol outcome variables (i.e., significant main effect cognitive measure). All independent variables were first zero-centered, and interaction terms were calculated by multiplying the cognitive measure with age group. The simple effects of a cognitive measure and age group were jointly entered in a first step and the interaction between these two variables was entered in the second step. A bootstrapped approach $(k=5000)$ with $95 \%$ confidence intervals was used to account for potential violations in distributional assumptions.

Given the novelty of the age group comparison and to allow for novel hypothesis formation, we decided not to correct for multiple comparisons. Instead, we complemented the frequentist analysis with Bayesian analysis to quantify evidence for the null hypothesis. Bayes factors (BF) are reported representing the probability of our data under the $\mathrm{H} 0$ relative to $\mathrm{H} 1$ with default priors (BF01). For interpretation of the evidence strength we followed Jeffreys [33]; Anecdotal (i.e., not enough evidence to support or refute $\mathrm{H} 0)=\mathrm{BF} 1-3$, moderate $=\mathrm{BF} 3-10$, strong $=\mathrm{BF} 10-30$, very strong $=\mathrm{BF} 30-100$, and extremely strong $=\mathrm{BF}>100$. Of note, evidence strength for $\mathrm{H} 1$ (i.e., BF10) can derived from 1/BF01. All analyses were run in JASP (JASP Team, 2019).

\section{Results}

\subsection{Group Comparison between Adolescents and Adults}

Total AUDIT (see Figure 1), AUDIT problems, average monthly alcohol use (standard units), and onset of alcohol use did not significantly differ between adolescents and adults, with moderate evidence levels (BF01 > 3, see Table 1). Despite similar levels of monthly alcohol use, the current data provided extremely strong evidence that for adolescents this amount was consumed over less occasions; adolescents reported significantly fewer drinking days per month $(\mathrm{U}=457.0, \mathrm{z}=4.54$, $\mathrm{p}<0.001, \mathrm{r}=0.68, \mathrm{BF} 01=0.007)$ and more standard drinks per drinking episode $(\mathrm{U}=427.0, \mathrm{z}=4.76$, $\mathrm{p}<0.001, \mathrm{r}=0.71, \mathrm{BF} 01=0.005)$. Some adolescents never binged $(\mathrm{n}=2)$ or had never been drunk $(n=6)$, but among those participants who did, age of first binge $(U=587.5, z=3.26, p=0.001, r=0.34$, $\mathrm{BF} 01=0.07)$ and age of first time drunk $(\mathrm{U}=646.5, \mathrm{z}=1.99, \mathrm{p}=0.047, \mathrm{r}=0.22, \mathrm{BF} 01=1.23)$ were significantly lower in adolescents compared to adults. Moreover, the current data provide anecdotal evidences for higher depressive symptoms (BDI; $\mathrm{U}=748.5, \mathrm{z}=2.14, \mathrm{p}=0.033, \mathrm{r}=0.23, \mathrm{BF} 01=0.43$ ) and lower lifetime illicit substance use $(\mathrm{U}=678.5, \mathrm{z}=2.54, \mathrm{p}=0.011, \mathrm{r}=0.28, \mathrm{BF} 01=0.70)$ in adolescents compared to adults. 
Regarding motivational and control measures, the current data provided very strong evidence for higher impulsivity (BIS-11; $\mathrm{t}=3.58, \mathrm{p}=0.001, \mathrm{~d}=0.76, \mathrm{BF} 01=0.02$ ) and moderate evidence for higher enhancement drinking motives (DMQ-r; $\mathrm{U}=675.5, \mathrm{z}=2.60, \mathrm{p}=0.009, \mathrm{r}=0.27, \mathrm{BF} 01=0.24$ ) in adolescents compared to adults. None of the other measures significantly differed between groups, with moderate evidence levels (BF01 $>3$, see Table 1). However, a non-parametric one-sample Wilcoxon signed-rank test showed that both adolescents and adults had a significant attentional bias (alcohol Stroop) towards alcohol (i.e., larger than zero, $\mathrm{z}=2.20, \mathrm{p}=0.028, \mathrm{r}=0.23$, BF01 $=0.17$ ). Regarding the alcohol approach bias (CA-AAT), there was no significant main effect of Bias Condition $\left(\mathrm{F}(3,85)=0.25, \mathrm{p}=0.86\right.$, partial $\left.\eta^{2}=0.003, \mathrm{BF} 01=59.26\right)$ and Group $(\mathrm{F}(1,87)=0.46, \mathrm{p}=0.50$, partial $\eta^{2}=0.005$, BF01 = 3.79), and no significant interaction between Bias Condition and Group $\left(\mathrm{F}(3,85)=0.36, \mathrm{p}=0.78\right.$, partial $\left.\eta^{2}=0.004, \mathrm{BF} 01=22.88\right)$, indicating that both age groups did not show a significant alcohol approach bias with moderate to very strong evidence levels.

\subsection{Associations of Alcohol Use Measures with Motivational and Control Processes}

Table 2 depicts the non-parametric Kendall's tau rank correlation coefficients and BF01 of the alcohol use measures with the motivational and control measures. AUDIT problems correlated weakly positively with monthly drinking days $(\mathrm{r} \tau=0.30, p<0.001, \mathrm{BF} 01=0.01)$ and drinks per episode $(\mathrm{r} \tau=0.25, p=0.002$, BF01 $=0.002)$, but monthly drinking days and drinks per episode did not correlate $(\mathrm{r} \tau=-0.14, p=0.09, \mathrm{BF} 01=0.78)$, supporting separate assessments of these variables in further analyses. There was very to extremely strong evidence for a weak to moderately positive correlation of AUDIT problems with social $(\mathrm{r} \tau=0.26, p<0.001$, BF01 $=0.014)$, coping $(\mathrm{r} \tau=0.29, p<0.001$, BF01 $=0.002)$, and enhancement drinking motives $(r \tau=0.41, p<0.001$, BF01 < 0.0001). Similarly, there was moderate to very strong evidence for a weak to moderate positive correlation of monthly drinking days with social $(\mathrm{r} \tau=0.24, p<0.001$, BF01 $=0.04)$, coping $(\mathrm{r}=0.29, p<0.001$, BF01 $=0.002)$, and enhancement drinking motives $(\mathrm{r} \tau=0.21, p<0.001, \mathrm{BF} 01=0.13)$. Drinks per episode correlated weakly positively with social $(\mathrm{r} \tau=0.19, p=0.015$, BF01 $=0.26)$ and enhancement $(\mathrm{r} \tau=0.37, p<0.001$, BF01 < 0.0001) drinking motives, with moderate to extremely strong evidence. Impulsivity correlated weakly positively with AUDIT problems $(\mathrm{r} \tau=0.17, p=0.027, \mathrm{BF} 01=0.47)$ and drinks per drinking episode $(\mathrm{r} \tau=0.15, p=0.045$, BF01 $=0.79$ ), with only anecdotal evidence. Evidence was generally moderate for the absence of a correlation between alcohol use and the other cognitive measures (i.e., BF01 > 3, see Table 2). However, the current data was insufficient to support or reject a relation between drinks per drinking episode and the attentional and approach bias, monthly drinking days and craving, and conformity drinking motives and AUDIT problems and monthly drinking days.

Table 2. Univariate association between alcohol use, motivational, and control measures.

\begin{tabular}{|c|c|c|c|c|c|c|}
\hline \multirow[t]{2}{*}{ Measure } & \multicolumn{2}{|c|}{ AUDIT Problems } & \multicolumn{2}{|c|}{$\begin{array}{l}\text { Monthly Use } \\
\text { (Drinking Days) }\end{array}$} & \multicolumn{2}{|c|}{$\begin{array}{c}\text { Drinks per Drinking } \\
\text { Episode (Standard Units) }\end{array}$} \\
\hline & $\mathbf{r} \tau$ & $\mathrm{BF}_{01}$ & $\mathbf{r \tau}$ & $\mathrm{BF}_{01}$ & $\mathbf{r \tau}$ & $\mathrm{BF}_{01}$ \\
\hline Session induced alcohol craving (VAS) & 0.08 & 4.06 & 0.11 & 2.10 & -0.06 & 5.44 \\
\hline Alcohol attentional bias (Alcohol Stroop) & -0.01 & 7.10 & -0.02 & 6.96 & -0.12 & 1.94 \\
\hline Alcohol approach bias (CA-AAT, ms) & -0.08 & 3.90 & 0.08 & 3.92 & -0.14 & 1.05 \\
\hline Social drinking motives (DMQ-r) & $0.26^{* *}$ & 0.01 & $0.24 *$ & 0.04 & $0.19 *$ & 0.26 \\
\hline Coping drinking motives (DMQ-r) & $0.29 * *$ & 0.00 & $0.29 * *$ & 0.00 & 0.04 & 6.06 \\
\hline Enhancement drinking motives (DMQ-r) & $0.41^{* *}$ & 0.00 & $0.21 *$ & 0.13 & $0.37 * *$ & 0.00 \\
\hline Conformity drinking motives (DMQ-r) & 0.15 & 0.78 & 0.13 & 1.68 & -0.08 & 3.78 \\
\hline Impulsivity (BIS-11) & $0.17 *$ & 0.47 & -0.06 & 5.32 & $0.15 *$ & 0.79 \\
\hline Interference control (Classical Stroop) & 0.08 & 3.87 & 0.01 & 7.18 & -0.03 & 6.53 \\
\hline Risky decision making (CCT) & -0.01 & 7.21 & -0.02 & 6.90 & -0.02 & 7.01 \\
\hline Working memory (SOPT) & -0.02 & 6.84 & 0.07 & 4.37 & 0.01 & 7.06 \\
\hline
\end{tabular}

Results of non-parametric Kendall's Tau rank correlations (r) and Bayes factor likelihood H0 relative to H1 with default priors $\left(\mathrm{BF}_{01}\right){ }^{*} p<0.05,{ }^{* *} p<0.001$; AUDIT: Alcohol Use Disorder Identification Test; VAS: Visual Analogue Scale; CA-AAT: Cannabis Alcohol Approach Avoidance Task; DMQ-r: Drinking Motives Questionnaire Revised; BIS-11: Barratt Impulsiveness Scale; CCT: Colombia Card Task; SOPT: Self-ordered Pointing task. 


\subsection{Does Age Moderate the Relation between Alcohol Use and Cognition?}

Only impulsivity, and social, coping, and enhancement drinking motives correlated with alcohol use. For these cognitive measures and the specific alcohol use measure they correlated with, we investigated if age moderated the relationship. Impulsivity correlated weakly with social, coping, and enhancement drinking motives ( $\mathrm{r} \tau=0.16-0.26$, ps $<0.032)$, while social, coping and enhancement correlated weakly to moderately with each other $(\mathrm{r} \tau=0.28-0.48, p<0.001)$. Given these weak to moderate associations, we first conducted separate moderation analyses, but planned to conduct a combined analysis to assess individual contribution of each predictor in case of multiple significant moderation effects for a given dependent variable. Results of the bootstrapped moderation analyses and BF01 of including the interaction between cognitive measure and age to the null model including the main effects of cognitive measure and age are shown in Table 3. Age only moderated the association between social drinking motives and AUDIT problems $\left(\Delta \mathrm{R}^{2}=0.05, \mathrm{~b}=0.34, p=0.04\right.$, BF01 $=0.69)$, with a stronger positive association in adolescents $(\mathrm{r} \tau=0.39)$ compared to adults $(\mathrm{r} \tau=0.11)$. However, the evidence was anecdotal (BF01 close to 1) and the B confidence interval contained 0, reducing the confidence in this finding. None of the other interactions between age and cognition significantly explained variance in alcohol use. Evidence for the absence of a moderating effect of age in these analyses was anecdotal to moderate (BF01 between 1.13-3.86).

Table 3. Outcomes moderation analyses.

\begin{tabular}{|c|c|c|c|c|c|c|c|c|}
\hline Predictor & Alcohol Use Outcome & $\Delta R^{2}$ & B & $95 \%$ CI bca (B) & SE (B) & $b$ & $p$ & $\mathrm{BF}_{01}$ \\
\hline \multirow[t]{3}{*}{ Impulsivity (BIS-11) $\mathrm{X}$ age } & AUDIT problems & 0.01 & 0.55 & $-7.23-1.81$ & 0.65 & 0.13 & 0.45 & 1.43 \\
\hline & Monthly use (drinking days) & 0.03 & 2.83 & $-0.47-5.45$ & 1.49 & 0.30 & 0.05 & 0.59 \\
\hline & $\begin{array}{l}\text { Drinks per drinking episode } \\
\text { (standard units) }\end{array}$ & 0.01 & 0.52 & $-0.47-1.51$ & 0.50 & 0.13 & 0.29 & 2.50 \\
\hline \multicolumn{9}{|l|}{ Drinking motives (DMQ-r) } \\
\hline Social $\mathrm{X}$ age & AUDIT problems & 0.05 & 1.31 & $-0.26-4.14$ & 1.09 & 0.34 & 0.04 & 0.69 \\
\hline \multirow[t]{2}{*}{ Coping $X$ age } & AUDIT problems & 0.00 & 0.14 & $-1.03-1.96$ & 0.73 & 0.05 & 0.79 & 2.05 \\
\hline & Monthly use (drinking days) & 0.01 & -1.16 & $-4.37-1.59$ & 1.51 & -0.13 & 0.38 & 2.81 \\
\hline \multirow[t]{2}{*}{ Enhancement $X$ age } & AUDIT problems & 0.01 & 0.39 & $-0.84-3.26$ & 0.95 & 0.12 & 0.47 & 3.10 \\
\hline & Monthly use (drinking days) & 0.01 & -1.82 & $-4.13-0.98$ & 1.28 & -0.20 & 0.15 & 1.80 \\
\hline
\end{tabular}

CI bca: Confidence Interval bias corrected accelerated; SE: Standard Error; AUDIT problems: sum Alcohol Use Disorder Identification Test items 4-10; DMQ-r: Drinking Motives Questionnaire-revised; B, SE(B), 95\% CI are based on bootstrapping results of 5000 replications. $\mathrm{BS}_{01}$ : Bayes factor likelihood $\mathrm{H} 0$ relative to $\mathrm{H} 1$ with default priors of including cognition $\mathrm{X}$ age to null model including cognitive measure and age.

\section{Discussion}

The current study aimed to unravel the moderating role of age in the relation between alcohol use and different motivational and control processes in adolescent drinkers compared to adults. Clear strengths of this study include the exploration of a wide range of cognitive measures with a frequentist and Bayesian approach and the direct comparison of adolescent and adult drinkers that were closely matched on monthly alcohol (drinks per month) consumption and alcohol use-related problems. Three different aspects of alcohol use were investigated: severity of alcohol use-related problems, drinking days per month, and drinks per drinking episode. The current data provide extremely strong evidence for more binge-drinking like behaviors, very strong evidence for higher impulsivity, and moderate evidence for higher enhancement drinking motives (i.e., drinking for positive affect) in adolescents compared to adults. Impulsivity and enhancement drinking motives also related positively to alcohol use, but independently of age. Coping and social drinking motives also related positively with alcohol use with some anecdotal evidence that the relation between social drinking motives and severity of alcohol use-related problems is stronger in adolescents. However, none of the other 
alcohol use-related motivational measures (i.e., craving, attentional bias, and approach bias) and behavioral control measures (i.e., interference control, risky decision making, and working-memory) were associated with alcohol use or differed between age groups. These findings support the role of elevated impulsivity and affective sensitivity in adolescent AUD risk, but question the role of craving, attentional bias, approach-bias, and cognitive control $[13,19,20]$, and the moderating role of age therein. A detailed discussion of these findings is provided below.

Impulsivity as measured with the BIS-11 [29] was higher in adolescents and positively related to alcohol use-related problems and drinks per drinking episode across age groups, but not to drinking days per month. Even though the evidence for a relation between impulsivity and alcohol use was small and anecdotal in the present study, the association between BIS-11 and various aspects of use is well replicated in light to heavy drinking adolescents and adults e.g., [34-37]. Importantly, impulsivity is a complex multifaceted construct, and the BIS-11 is a self-report measure that typically poorly correlates with behavioral laboratory tasks of impulse control [38]. Although the nature and distinctiveness of different impulsivity components is part of current debate $[39,40]$, the role of different impulsivity components is thought to vary over the course of alcohol use towards dependence [41,42]. Impulsivity is thought to peak during adolescence and individuals in which impulsivity declines more slowly towards adulthood are thought to be at a higher risk for increasing their alcohol use [43]. Moreover, impulsivity is thought to be both causally and consequentially related to addiction, interacting with many other addiction risk factors [44,45], including coping and enhancement drinking motives in the current study. These findings suggest a complex but developmentally sensitive role of impulsivity in alcohol use and predict lower levels of impulsivity in non-drinking adolescents. We only included drinking adolescents and adults. To further investigate these issues, more fine-grained longitudinal investigation including different behavioral and self-report measures of impulsivity, in both drinking and non-drinking groups are needed.

The four drinking motives defined by Cooper [28] pertain to the valence (positive or negative reinforcement) and the source (internally or externally driven) of drinking outcomes; people drink to enhance positive affect, to cope with negative feelings, to increase social connections, or to conform to social group norms and avoid peer rejections. A long line of research into drinking motives shows that adolescents and adults mostly drink for social reasons but the severity of alcohol use-related problems mainly relates to enhancement and coping motives [46]. Moreover, decreases in alcohol use-related problems over the course of adolescence towards adulthood relate to decreases in enhancement and coping motives [47]. Changes in drinking motives over time may thereby relate to AUD risk during adolescence, but resilience during emerging adulthood. The current data provided extremely strong support of a moderately positive relation of social, coping, and enhancement motives with alcohol use-related problems. While social motives were most prevalent, coping motives were generally low and only enhancement motives were higher in adolescents compared to adults. Considering the potential moderating role of age, the current data mostly provided anecdotal evidence (see Table 3), preventing us from drawing firm conclusions in this regard. A post hoc explorative multiple regression analysis including all three motives indicated that only enhancement motives explained unique variance in problem severity $[\mathrm{B}=0.30,95 \% \mathrm{CI}(\mathrm{B})=0.13-0.45, p=0.001]$. These findings support a general role for enhancement, but not for coping, social, and conformity motives in the severity of drinking across the current sample of adolescents and adults.

Based on primarily theoretical support $[13,20]$, alcohol craving, attentional bias, and approach bias were expected to be higher in adolescents but to relate to alcohol use irrespective of age. The results of the current study did not confirm this. Although both groups had an attentional bias towards alcohol, the current data provided moderate evidence that none of the alcohol oriented motivational measures differed between adolescents and adults. There was also moderate evidence for the absence of a correlation with alcohol use-related problems. Importantly however, our data was insufficient to support of refute a relation between drinks per drinking episode and the attentional and approach bias, and monthly drinking days and craving. While there is a general paucity of studies, two previous 
studies also did not find an age difference in craving and attentional bias between adolescents and adults $[16,48]$. Although there is substantial support for the role of approach bias in addiction, the generally poor reliability of the AAT and related measures is a known issue [27] that may explain the lack of an approach bias and a relation with alcohol use, and warrants the development of more reliable measures. Nevertheless, craving, attentional bias, and approach bias are expected to selectively emerge in heavy and dependent users. Based on the AUDIT scores, half of the participants were "at-risk" drinkers ([22]; see Figure 1). Inclusion of more frequent and dependent drinkers should therefore be considered in future studies.

While human studies investigating the role of age in the relation between alcohol use and cognitive control are missing, there is evidence from rodent studies to suggest that adolescent compared to adult onset drinkers experience stronger reductions in cognitive control-related functions (for reviews see [49,50]), including more extensive prefrontal cortex damage [51] and increases in impulsive decision making [52]. Moreover, adolescent animals appear less prone to withdrawal [53], and yet also less prone to tolerance [54], impairments in behavioral flexibility [55], and for loss of control over alcohol use [56]. These findings highlight the complex relationship between adolescent alcohol exposure and AUD-like behaviors in later life; exposure to alcohol during adolescence may render individuals more sensitive to some effects of alcohol, but less sensitive to others. The current data provided moderate evidence that adolescents and adults performed equally on the Stroop, CCT, and SOPT, cognitive control-related tasks that respectively aimed to measure interference control, risky decision making and working memory. Moreover, performance on these tasks did not relate to alcohol use. Of note, for most cognitive functions, the strongest development is seen before adolescence [57]. Improvement in interference control as measured with the Classical Stroop test and risky decision making, as measured with the CCT, between the age of 16 to 35 is generally subtle [31,58]), which may explain the lack of a significant difference between our age groups. Working memory refers to maintenance, updating, and manipulation of online information, which is a central cognitive function in which many other cognitive functions come together [59]. Supporting construct validity, SOPT performance correlated positively with STROOP $(r=0.18)$ and negatively with CCT performance $(r=-0.23)$. Nevertheless, the SOPT is a relatively simple task that does not require information manipulation, which potentially explains the lack of a developmental difference. Moreover, relative insensitivity of the tasks to developmental changes between late adolescence and adulthood also hinders the detection of potential moderating effects of age on the relation between cognition and alcohol use. In the context of addiction vulnerability and adolescent to adult development [13], future studies are therefore recommended to specifically develop and include tasks that tap into the interplay between emotion regulation, affective reactivity, and behavioral control, rather than assessing them as separate constructs. Furthermore, highlighted by the prevalence of social drinking motives, the moderating role of age on alcohol related cognitions may only be visible in a social context. While human studies on this topic are missing, animal studies support the differential relation between social cognition and alcohol use in adolescent versus adult rats [60].

Some limitations should be considered. Firstly, age directly relates to quantity and duration of alcohol exposure (i.e., adults have been drinking for a longer period of time). Although the absence of a significant age group difference for all cognitive tasks suggests a minimal role of duration of use, the current design cannot control for the differences in alcohol use history between adolescents and adults. Moreover, it is recommended to include non-drinking adolescents and adults in future studies to distinguish effects of lifetime alcohol use (yes/no) from age group effects. Secondly, animal work suggests distinct vulnerability periods across adolescence, with higher vulnerability if alcohol use is started in early adolescence [15]. We tested older adolescents. Since on average both adolescents and adults started to drink at the age of 14, a moderating role of age may then only be visible in even younger age groups, stressing the need for testing wider age-ranges. Thirdly, the cross-sectional approach precludes causal inferences, for which either longitudinal studies before the onset of alcohol use or animal studies with direct control over alcohol exposure are needed. Fourthly, our study was 
insufficiently powered to detect small effects. Finally, as discussed above, the included motivational and control measures cover a limited part of functioning and the generalizability of the current results to other cognitive domains remains to be tested.

\section{Conclusions}

Despite similar levels of alcohol use-related problems and monthly alcohol use, adolescents showed more binge-like patters of alcohol use. The current data did not support a moderating role of age in the relation between alcohol use and cognition. However, the findings suggest an important role for impulsivity and enhancement drinking motives in adolescent alcohol use specifically, due to their elevated levels in adolescents compared to adults and their consistent relation with different alcohol measures. The current study therefore provides an important step towards understanding the role of age on the impact of alcohol on brain and behavior. Nevertheless, a replication and extension to other cognitive domains and age ranges is warranted to further clarify the relation between age, cognition, and AUDs.

Author Contributions: J.C., H.M.B.L., L.J.M.J.V., and J.L.K. designed the study. J.C., H.M.B.L., and L.J.M.J.V. acquired funding. K.H.G. and M.L. collected the data. J.C. and K.H.G. analyzed the data and J.C. drafted the manuscript. All authors reviewed and edited the manuscript. All authors have read and agreed to the published version of the manuscript.

Funding: This work was supported by a seed grant from the Utrecht University Strategic Theme Dynamics of Youth and an Amsterdam Brain and Cognition project grant.

Conflicts of Interest: The authors declare no conflict of interest.

\section{References}

1. Crone, E.A.; Dahl, R.E. Understanding adolescence as a period of social-affective engagement and goal flexibility. Nat. Rev. Neurosci. 2012, 13, 636-650. [CrossRef] [PubMed]

2. Chassin, L.; Fora, D.B.; King, K.M. Trajectories of alcohol and drug use and dependence from adolescence to adulthood: The effects of familial alcoholism and personality. J. Abnorm Psychol. 2004, 113, 483-498. [CrossRef] [PubMed]

3. Lee, M.R.; Boness, C.L.; McDowell, Y.E.; Vergés, A.; Steinley, D.L.; Sher, K.J. Desistance and Severity of Alcohol Use Disorder: A Lifespan-Developmental Investigation. Clin. Psychol. Sci. 2018, 6, 90-105. [CrossRef] [PubMed]

4. Johnston, L.D.; Miech, R.A.; O'Malley, P.M.; Bachman, J.G.; Schulenberg, J.E.; Patrick, M.E. Monitoring the Future National Survey Results on Drug Use, 1975-2017: Overview key findings on adolescent drug use 2018. Available online: https:/files.eric.ed.gov/fulltext/ED589762.pdf (accessed on 21 September 2020).

5. Carroll, L.J.; Cassidy, J.D.; Peloso, P.M.; Borg, J.; von Holst, H.; Holm, L.; Paniak, C.; Pepin, M. Prognosis for mild traumatic brain injury: Results of the WHO Collaborating Centre Task Force on Mild Traumatic Brain Injury. J. Rehabil Med. 2004, 84-105. [CrossRef] [PubMed]

6. Uhl, G.R.; Koob, G.F.; Cable, J. The neurobiology of addiction. Ann. N. Y. Acad. Sci. 2019, 62, 118-127. [CrossRef]

7. Asbridge, M.; Cartwright, J.; Wilson, K.; Langille, D. Age at first drink, experiences of drunkenness, and alcohol-related problems in Canadian youth: Is early onset bad if you are a moderate drinker? J. Stud. Alcohol Drugs 2016, 77, 974-979. [CrossRef]

8. DeWit, D.J.; Adlaf, E.M.; Offord, D.R.; Ogborne, A.C. Age at first alcohol use: A risk factor for the development of alcohol disorders. Am. J. Psychiatry 2000, 157, 745-750. [CrossRef]

9. Liang, W.; Chikritzhs, T. Age at first use of alcohol and risk of heavy alcohol use: A population-based study. Biomed Res. Int. 2013, 2013. [CrossRef]

10. Pitkänen, T.; Lyyra, A.L.; Pulkkinen, L. Age of onset of drinking and the use of alcohol in adulthood: A follow-up study from age $8-42$ for females and males. Addiction 2005, 100, 652-661. [CrossRef]

11. Steinberg, L. Cognitive and affective development in adolescence. Trends Cogn. Sci. 2005, 9, 69-74. [CrossRef]

12. Davidow, J.Y.; Foerde, K.; Galván, A.; Shohamy, D. An upside to reward sensitivity: The hippocampus supports enhanced reinforcement learning in adolescence. Neuron 2016, 92, 93-99. [CrossRef] [PubMed] 
13. Cousijn, J.; Luijten, M.; Feldstein Ewing, S.W. Adolescent resilience to addiction: A social plasticity hypothesis. Lancet Child Adolesc. Heal. 2018, 2, 69-78. [CrossRef]

14. Squeglia, L.M.; Jacobus, J.; Tapert, S.F. The effect of alcohol use on human adolescent brain structures and systems. In Handbook of Clinical Neurology; Elsevier: Edinburgh, UK, 2014; Volume 125, pp. 501-510.

15. Spear, L.P. Adolescent alcohol exposure: Are there separable vulnerable periods within adolescence? Physiol. Behav. 2015, 148, 122-130. [CrossRef] [PubMed]

16. McAteer, A.M.; Hanna, D.; Curran, D. Age-related differences in alcohol attention bias: A cross-sectional study. Psychopharmacology 2018, 235, 2387-2393. [CrossRef]

17. Scheel, J.F.; Schielke, K.; Lautenbacher, S.; Aust, S.; Kremer, S.; Wolstein, J. Low-Dose Alcohol Effects on Attention in Adolescents. Zeitschrift für Neuropsychol. 2013, 24, 103-111. [CrossRef]

18. Rooke, S.E.; Hine, D.W. A dual process account of adolescent and adult binge drinking. Addict. Behav. 2011, 36, 341-346. [CrossRef] [PubMed]

19. Wiers, R.W.; Bartholow, B.D.; Van den Wildenberg, E.; Thush, C.; Engels, R.C.M.E.; Sher, K.J.; Grenard, J.; Ames, S.L.; Stacy, A.W. Automatic and controlled processes and the development of addictive behaviors in adolescents: A review and a model. Pharmacol. Biochem. Behav. 2007, 86, 263-283. [CrossRef] [PubMed]

20. Conrod, P.; Nikolaou, K. Annual Research Review: On the developmental neuropsychology of substance use disorders. J. Child Psychol. Psychiatry 2016, 57, 371-394. [CrossRef]

21. Saunders, J.B.; Aasland, O.G.; Babor, T.F.; de la Fuente, J.R.; Grant, M. Development of the Alcohol Use Disorders Identification Test (AUDIT): WHO Collaborative Project on Early Detection of Persons with Harmful Alcohol Consumption-II. Addiction 1993, 88, 791-804. [CrossRef]

22. Babor, T.F.; Robaina, K. The Alcohol Use Disorders Identification Test (AUDIT): A review of graded severity algorithms and national adaptations. Int. J. Alcohol Drug Res. 2016, 5, 17. [CrossRef]

23. Heatherton, T.F.; Kozlowski, L.T.; Frecher, R.C.; Fagerstrom, K.O. The Fagerström Test for Nicotine Dependence: A revision of the Fagerstrom Tolerance Questionnaire. Br. J. Addict. 1991, 86, 1119-1127. [CrossRef] [PubMed]

24. Beck, A.T.; Steer, R.A.; Ball, R.; Ranieri, W.F. Comparison of Beck depression inventories -IA and -II in psychiatric outpatients. J. Pers. Assess. 1996, 67, 588-597. [CrossRef] [PubMed]

25. Spielberger, C.D.; Gorsuch, R.L.; Lushene, R.E. The State Trait Anxiety Inventory; Consulting Psychologists Press: Palo Alto, CA, USA, 1970.

26. Cousijn, J.; van Benthem, P.; van der Schee, E.; Spijkerman, R. Motivational and control mechanisms underlying adolescent cannabis use disorders: A prospective study. Dev. Cogn. Neurosci. 2015, 16, 36-45. [CrossRef] [PubMed]

27. Ataya, A.F.; Adams, S.; Mullings, E.; Cooper, R.M.; Attwood, A.S.; Munafò, M.R. Internal reliability of measures of substance-related cognitive bias. Drug Alcohol Depend. 2012, 121, 148-151. [CrossRef]

28. Cooper, M.L. Motivations for alcohol use among adolescents: Development and validation of a four-factor model. Psychol. Assess. 1994, 6, 117-128. [CrossRef]

29. Patton, J.H.; Stanford, M.S.; Barratt, E.S. Factor structure of the barratt impulsiveness scale. J. Clin. Psychol. 1995, 51, 768-774. [CrossRef]

30. Hammes, J.G.W. De Stroop Kleur-Woord Test Handleiding; Swets en Zeitlinger: Lisse, The Netherlands, 1971.

31. Figner, B.; Mackinlay, R.J.; Wilkening, F.; Weber, E.U. Affective and Deliberative Processes in Risky Choice: Age Differences in Risk Taking in the Columbia Card Task. J. Exp. Psychol. Learn. Mem. Cogn. 2009, 35, 709-730. [CrossRef]

32. Petrides, M.; Milner, B. Deficits on subject-ordered tasks after frontal- and temporal-lobe lesions in man. Neuropsychologia 1982, 20, 249-262. [CrossRef]

33. Jeffreys, H. Theory of Probability 3rd edition, 3rd ed.; Clarendon Press: Oxford, UK, 1961.

34. Carlson, S.R.; Johnson, S.C. Impulsivity is not always associated with student drinking: A moderation study of impulsivity and drinking by positive alcohol expectancies. Addict. Behav. 2012, 37, 556-560. [CrossRef]

35. Fernie, G.; Cole, J.C.; Goudie, A.J.; Field, M. Risk-taking but not response inhibition or delay discounting predict alcohol consumption in social drinkers. Drug Alcohol Depend. 2010, 112, 54-61. [CrossRef]

36. Goudriaan, A.E.; Grekin, E.R.; Sher, K.J. Decision making and binge drinking: A longitudinal study. Alcohol. Clin. Exp. Res. 2007, 31, 928-938. [CrossRef] 
37. Von Diemen, L.; Bassani, D.G.; Fuchs, S.C.; Szobot, C.M.; Pechansky, F. Impulsivity, age of first alcohol use and substance use disorders among male adolescents: A population based case-control study. Addiction 2008, 103, 1198-1205. [CrossRef] [PubMed]

38. Sharma, L.; Markon, K.E.; Clark, L.A. Toward a theory of distinct types of "impulsive" behaviors: A meta-analysis of self-report and behavioral measures. Psychol. Bull. 2014, 140, 374-408. [CrossRef] [PubMed]

39. Dalley, J.W.; Robbins, T.W. Fractionating impulsivity: Neuropsychiatric implications. Nat. Rev. Neurosci. 2017, 18, 158-171. [CrossRef]

40. Fineberg, N.A.; Chamberlain, S.R.; Goudriaan, A.E.; Stein, D.J.; Vanderschuren, L.J.M.J.; Gillan, C.M.; Shekar, S.; Gorwood, P.A.P.M.; Voon, V.; Morein-Zamir, S.; et al. New developments in human neurocognition: Clinical, genetic, and brain imaging correlates of impulsivity and compulsivity. CNS Spectr. 2014, 19, 69-89. [CrossRef] [PubMed]

41. Hershberger, A.R.; Um, M.; Cyders, M.A. The relationship between the UPPS-P impulsive personality traits and substance use psychotherapy outcomes: A meta-analysis. Drug Alcohol Depend. 2017, 178, 408-416. [CrossRef]

42. Leung, D.; Staiger, P.K.; Hayden, M.; Lum, J.A.G.; Hall, K.; Manning, V.; Verdejo-Garcia, A. Meta-analysis of the relationship between impulsivity and substance-related cognitive biases. Drug Alcohol Depend. 2017, 172, 21-33. [CrossRef]

43. Quinn, P.D.; Harden, K.P. Differential changes in impulsivity and sensation seeking and the escalation of substance use from adolescence to early adulthood. Dev. Psychopathol. 2013, 25, 223-239. [CrossRef]

44. Argyriou, E.; Um, M.; Carron, C.; Cyders, M.A. Age and impulsive behavior in drug addiction: A review of past research and future directions. Pharmacol. Biochem. Behav. 2018, 164, 106-117. [CrossRef]

45. De Wit, H. Impulsivity as a determinant and consequence of drug use: A review of underlying processes. Addict. Biol. 2009, 14, 22-31. [CrossRef]

46. Kuntsche, E.; Knibbe, R.; Gmel, G.; Engels, R. Why do young people drink? A review of drinking motives. Clin Psychol Rev 2005, 25, 841-861. [CrossRef] [PubMed]

47. Littlefield, A.K.; Sher, K.J.; Wood, P.K. Do changes in drinking motives mediate the relation between personality change and "maturing out" of problem drinking? J Abnorm Psychol 2010, 119, 93-105. [CrossRef]

48. Treloar Padovano, H.; Miranda, R. Subjective cannabis effects as part of a developing disorder in adolescents and emerging adults. J. Abnorm. Psychol. 2018, 127, 282-293. [CrossRef] [PubMed]

49. Spear, L.P. Effects of adolescent alcohol consumption on the brain and behaviour. Nat. Rev. Neurosci. 2018, 19, 197-214. [CrossRef] [PubMed]

50. Schramm-Sapyta, N.L.; Walker, Q.D.; Caster, J.M.; Levin, E.D.; Kuhn, C.M. Are adolescents more vulnerable to drug addiction than adults? Evidence from animal models. Psychopharmacology 2009, 206, 1-21. [CrossRef]

51. Crews, F.T.; Braun, C.J.; Hoplight, B.; Switzer, R.C.; Knapp, D.J. Binge ethanol consumption causes differential brain damage in young adolescent rats compared with adult rats. Alcohol. Clin. Exp. Res. 2000, 24, 1712-1723. [CrossRef]

52. Schindler, A.G.; Tsutsui, K.T.; Clark, J.J. Chronic alcohol intake during adolescence, but not adulthood, promotes persistent deficits in risk-based decision making. Alcohol. Clin. Exp. Res. 2014, 38, 1622-1629. [CrossRef]

53. Lee, K.M.; Coelho, M.A.; McGregor, H.A.; Solton, N.R.; Cohen, M.; Szumlinski, K.K. Adolescent Mice Are Resilient to Alcohol Withdrawal-Induced Anxiety and Changes in Indices of Glutamate Function within the Nucleus Accumbens. Front. Cell. Neurosci. 2016, 10. [CrossRef]

54. Pautassi, R.M.; Godoy, J.C.; Molina, J.C. Adolescent rats are resistant to the development of ethanol-induced chronic tolerance and ethanol-induced conditioned aversion. Pharmacol. Biochem. Behav. 2015, 138, 58-69. [CrossRef]

55. Fernandez, G.M.; Lew, B.J.; Vedder, L.C.; Savage, L.M. Chronic intermittent ethanol exposure leads to alterations in brain-derived neurotrophic factor within the frontal cortex and impaired behavioral flexibility in both adolescent and adult rats. Neuroscience 2017, 348, 324-334. [CrossRef]

56. Labots, M.; Cousijn, J.; Jolink, L.A.; Leon Kenemans, J.; Vanderschuren, L.J.M.J.; Lesscher, H.M.B. Age-related differences in alcohol intake and control over alcohol seeking in rats. Front. Psychiatry 2018, 9. [CrossRef] [PubMed] 
57. Waber, D.P.; De Moor, C.; Forbes, P.W.; Almli, C.R.; Botteron, K.N.; Leonarf, G.; Molovan, D.; Paus, T.; Rumsey, J. The NIH MRI study of normal brain development: Performance of a population based sample of healthy children aged 6 to 18 years on a neuropsychological battery. J. Int. Neuropsychol. Soc. 2007, 13, 729-746. [CrossRef] [PubMed]

58. Comalli, P.E.; Wapner, S.; Werner, H. Interference effects of stroop color-word test in childhood, adulthood, and aging. J. Genet. Psychol. 1962, 100, 47-53. [CrossRef] [PubMed]

59. Baddeley, A. Working Memory: Theories, Models, and Controversies. Annu. Rev. Psychol. 2012, 63, 1-29. [CrossRef]

60. Varlinskaya, E.I.; Spear, L.P. Acute Effects of Ethanol on Social Behavior of Adolescent and Adult Rats: Role of Familiarity of the Test Situation. Alcohol. Clin. Exp. Res. 2002, 26, 1502-1511. [CrossRef]

C 2020 by the authors. Licensee MDPI, Basel, Switzerland. This article is an open access article distributed under the terms and conditions of the Creative Commons Attribution (CC BY) license (http://creativecommons.org/licenses/by/4.0/). 